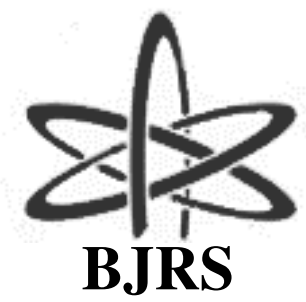

BRAZILIAN JOURNAL

$\mathrm{OF}$

RADIATION SCIENCES

03-1A (2015) 01-08

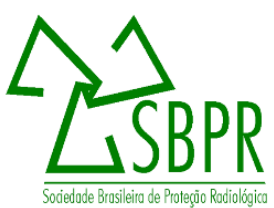

\title{
Avaliação do controle de qualidade de radiofármacos em serviço de medicina nuclear
}

\author{
Jamille A. Lopes Tavares ${ }^{\text {a }}$; Marcus Aurélio P. dos Santos ${ }^{\text {b }}$, Renata F. de Lira ${ }^{\mathrm{a}}$ \\ ${ }^{a}$ Universidade Federal de Pernambuco (UFPE) Av. Professor Moraes Rego, nº 1235 - CDU \\ 50670-901 Recife, PE \\ jam_alt@hotmail.com \\ renatafariasdelira@hotmail.com \\ ${ }^{\mathrm{b}}$ Centro Regional de Ciências Nucleares do Nordeste (CRCN-NE) Av. Professor Luiz Freire, nº 200 - CDU. \\ 50740-540 Recife, PE \\ masantos@cnen.gov.br
}

\begin{abstract}
Os radiofármacos são um tipo de preparação farmacêutica associada à radionuclídeos com finalidade diagnóstica e terapêutica. Serviços de Medicina Nuclear (SMN) devem realizar controle de qualidade dos radiofármacos conforme recomendações dos fabricantes e evidências científicas aceitas pela Agência Nacional de Vigilância Sanitária (ANVISA). Este trabalho avaliou a qualidade dos principais radiofármacos em um SMN do estado de Pernambuco em relação à pureza radioquímica e $\mathrm{pH}$. Os resultados mostraram que $96,8 \%$ dos radiofármacos apresentaram pureza radioquímica eficaz e que todos os valores de $\mathrm{pH}$ estavam dentro do intervalo recomendado pelas farmacopéias americanas. $\mathrm{O}$ estudo revelou que o controle de qualidade, quando inserido no SMN, fornece dados importantes que permite a exclusão de radiofármacos com baixa pureza radioquímica, favorecendo um diagnóstico confiável e garantindo boas práticas de radiproteção e de biossegurança para paciente e indivíduos ocupacionalmente expostos.
\end{abstract}

Keywords:Radiofármacos;Medicia Nuclear; Controle de qualidade.

\section{INTRODUCTION}

A Agência Nacional de Vigilância Sanitária (ANVISA) definiu radiofármacos como: "preparações farmacêuticas com finalidade diagnostica ou terapêutica que, quando prontas para 
o uso, contem um ou mais radionuclídeos, incluindo componentes extraídos dos geradores de radionuclídeos". A sua preparação necessita de uma seqüência de processos realizados em instalações apropriadas, seguindo um rígido programa de qualidade para garantir a pureza, esterilidade, pirogenicidade e estabilidade das preparações [1].

No Brasil, os radiofármacos foram isentos de regulamentação farmacêutica adequada por bastante tempo. A partir de 2008, a ANVISA, por meio de sua Resolução de Diretoria Colegiada (RDC) $n^{\circ} 38$, vem regulando este setor. A resolução dispõe que o Serviço de Medicina Nuclear (SMN) deve realizar o controle de qualidade do eluato dos geradores e radiofármacos conforme recomendação dos fabricantes, de evidencias cientifica ou de compêndios oficiais aceitos pela ANVISA. Desta forma, os SMN que não realizam os testes de controle de qualidade regulamentado pelas normas da ANVISA, não possuem informações reais da condição da qualidade dos radiofármacos no momento da administração ao paciente.

O Controle de Qualidade (CQ) é um conjunto de operações (programação, coordenação e execução) com o objetivo de verificar e assegurar que os produtos estão dentro do padrão de qualidade. O CQ consiste num dos pontos essenciais nas Normas de Boas Práticas Radiofarmacêuticas (BPR) para produtos farmacêuticos estéreis conforme recomendado pela Organização Mundial da Saúde (OMS). O CQ em SMN torna-se imprescindível uma vez que os radionuclídeos e radiofármacos são destinados para a administração em seres humanos.

Os testes de CQ estão divididos em duas categorias: físico-químicos e biológicos. Os testes físico-químicos indicam o nivel de purezas radionuclídicas e radioquímicas, determinação do $\mathrm{pH}$, forca iônica, osmolalidade, e estado físico da amostra. Os testes biológicos estabelecem a esterilidade, apirogenicidade e toxicidade do material [2]. Portanto, este trabalho propôs a avaliação da qualidade dos radiofármacos em um SMN do estado de Pernambuco em relação a pureza radioquímica e $\mathrm{pH}$ dos principais fármacos marcados com ${ }^{99 \mathrm{~m}} \mathrm{Tc}$ utilizados no setor.

\section{MATERIAIS E MÉTODOS}

Este trabalho foi realizado em um SMN de Recife, Pernambuco, no período de janeiro a dezembro de 2013. Durante o período do estudo foi realizado o levantamento do registro de marcação dos seguintes fármacos:

- 2-metoxi-2-isobutil isonitrila (MIBI);

- Metilenodifosfonado (MDP);

- Acido dimercaptosuccinico (DMSA);

- Acido dietilenotriamino pentacetico (DTPA) e

- Dihidrocloreto de bicisato (ECD).

Esses fármacos foram selecionados por serem os mais utilizados na marcação com ${ }^{99 \mathrm{~m}} \mathrm{Tc}$ na rotina desse SMN.

\subsection{Avaliação da Pureza Radioquímica}


A Pureza Radioquímica (PRQ) foi determinada por cromatografia de camada delgada seguindo o Protocolo Operacional Padrão (POP) do SMN. Esse procedimento tem o intuito de garantir a qualidade do radiofármaco a ser administrado no paciente, evitando remarcações de exames e, conseqüentemente, aumento na dose de radiação de pacientes e profissionais envolvidos na pratica medica.

A bula do fármaco informa as placas de cromatografia e os solventes a serem utilizados para verificar a eficiência do radiofármaco (tabela 1). Na fase estacionaria, foram empregadas placas cromatográficas de dimensões $1,0 \times 6,5 \mathrm{~cm}$. Cada placa possui linha de aplicação da amostra do radiofármaco, linha de corte e a linha superior. Na fase móvel, foram empregados solventes como descritos pelo fabricante.

Tabela 1: Recomendações para a análise cromatográfica do MIBI, MDP, DMSA, DTPA e ECD, de acordo com o controle de qualidade fornecido em bula pelo fabricante.

\begin{tabular}{llll} 
& FASE ESTACIONÁRIA & FASE MóvEL & PRQ(\%) \\
\hline MIBI & PLACA1-Papel Whatman & CUBA1-Metanol PA & $\geq 90 \%$ \\
& PLACA 2- Sílica Gel & CUBA2- NaCl 0,9\% & \\
MDP & PLACA 1 e PLACA 2 & CUBA1-Butonona PA & $\geq 85 \%$ \\
& Papel Whatman & CUBA2- NaCl 0,9\% & \\
DMSA & PLACA1-Papel Whatman & CUBA1-Butonona PA & $\geq 85 \%$ \\
& PLACA 2- Sílica Gel & CUBA2- NaCl 0,9\% & \\
DTPA & PLACA 1 e PLACA 2 & CUBA1-Butonona PA & $\geq 85 \%$ \\
& Papel Whatman & CUBA2- NaCl 0,9\% & \\
ECD & PLACA1-Papel Whatman & CUBA1-Butonona PA & $\geq 90 \%$ \\
& PLACA 2- Sílica Gel & CUBA2- NaCl 0,9\% &
\end{tabular}

Foram utilizadas duas placas de cromatografia para cada radiofármaco que, após aplicação da amostra em cada placa com o auxílio de uma pinça, foram colocadas em cubas cromatográficas com os solventes específicos, mantendo-se o ponto de aplicação para baixo e a fita na posição vertical. Após a absorção do solvente nas placas, as fitas de cromatografia foram retiradas das cubas cromatográficas com uma pinça e cortada cada uma delas na linha de corte. Utilizando um 
medidor de atividade (curiômetro), foi determinada a atividade radioativa de cada fração das respectivas placas cromatográficas. O resultado foi encontrado através da equação 1.

$$
\% \text { PRQ }=100-\left(\%{ }^{99 m} \mathrm{TcO}_{4}^{-}+\%{ }^{99} \mathrm{TcO}_{2}\right)
$$

\subsection{Avaliação do Potencial Hidrogeniônico (pH)}

$\mathrm{Na}$ avaliação do $\mathrm{pH}$ foi utilizado o papel indicador de $\mathrm{pH}$ de escala de 0 a 14 , fabricado pela Merck®. O radiofármaco foi gotejado na fita universal de $\mathrm{pH}$, realizando-se a leitura após 1 minuto (tempo mínimo recomendado pelo fabricante). A coloração obtida foi comparada à escala fornecida pelo fabricante. Os valores de referência do $\mathrm{pH}$ dos radiofármacos MIBI, MDP, DMSA, DTPA E ECD, segundo as bulas de fabricantes, estão descritos na Tabela 2.

Tabela 2: pH fornecido em bula pelo fabricante dos radiofármacos estudados.

\begin{tabular}{cc}
\cline { 2 - 2 } Radiofármacos & Faixa de pH \\
\hline MIBI & $5,0-6,0$ \\
MDP & $5,5-7,5$ \\
DMSA & $2,0-4,0$ \\
DTPA & $3,5-4,5$ \\
ECD & $6,0-8,0$ \\
\hline
\end{tabular}

\section{RESULTADOS E DISCUSSÕES}

\subsection{Avaliação da Pureza Radioquímica}

Os resultados da avaliação da PRQ estão representados na Figura 1. Foi observado, durante o estudo de cromatografia de camada delgada, que as 75 amostras de MIBI apresentaram uma PRQ (\%) igual ou superior fornecida pela bula do fabricante, conforme mostrada na Tabela 1. Isso ocorreu também com as 52 amostras de MDP, as 12 amostras de DTPA e as 10 amostras de ECD. Porém, das 43 amostras DMSA foi registrado que 6 amostras apresentam uma PRQ (\%) inferior ao informado pelo fabricante e não foram usadas na realização do exame. 
Figura 1: Valores de eficiência de marcação dos radiofármacos MIBI, MDP, DMSA, DTPA e ECD.

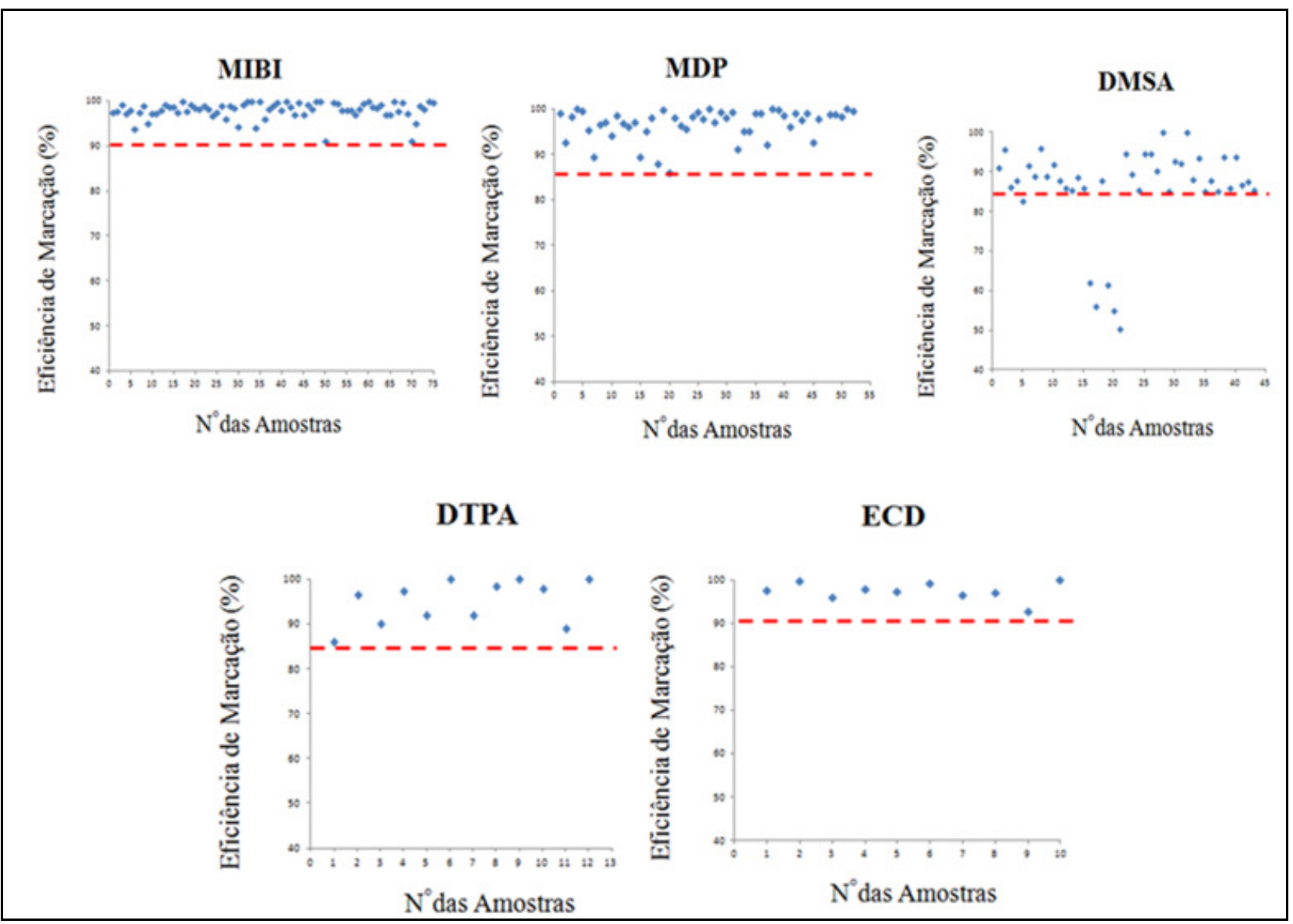

Com isso, comprova-se que o método de cromatografia em camada delgada é eficaz para avaliação e apresenta resultados capazes de determinar o descarte ou não da amostra em estudo. Os resultados desta avaliação foram registrados na ficha de Registro de Marcação de Fármacos do setor. Foi constatado que as amostras desmarcadas de DMSA estavam dentro do prazo de validade e o volume e a atividade administrada de ${ }^{99 \mathrm{~m}} \mathrm{Tc}$ estavam inferiores aos valores máximos informados na bula do fabricante. Porém, este SMN não realiza um controle de qualidade do eluato do gerador de ${ }^{99} \mathrm{Mo} /{ }^{99 \mathrm{~m}} \mathrm{Tc}$. Com isso, não se pode afirmar que a Eficiência de Marcação não atingiu um valor satisfatório devido ao ${ }^{99 \mathrm{~m}} \mathrm{Tc}$, aos erros durante o processo da marcação ou à produção do kit de DMSA fornecido pelo fabricante.

O uso de radiofármacos com especificações em desacordo ao que é recomendado por farmacopéias internacionais pode acarretar em formações de imagens inadequadas e repetições 
de exames diagnósticos, gerando custos materiais [3] e nova exposição às radiações ionizantes tanto do paciente quanto do trabalhador [4].

\subsection{Avaliação do Potencial Hidrogeniônico (pH)}

Na Figura 2 estão apresentados os valores de pH dos radiofármacos. Os resultados foram dispostos em gráficos com os limites propostos nas bulas dos fabricantes e farmacopéias internacionais.

Figura 2: Valores de pH dos radiofármacos MIBI, MDP, DMSA, DTPA e ECD.

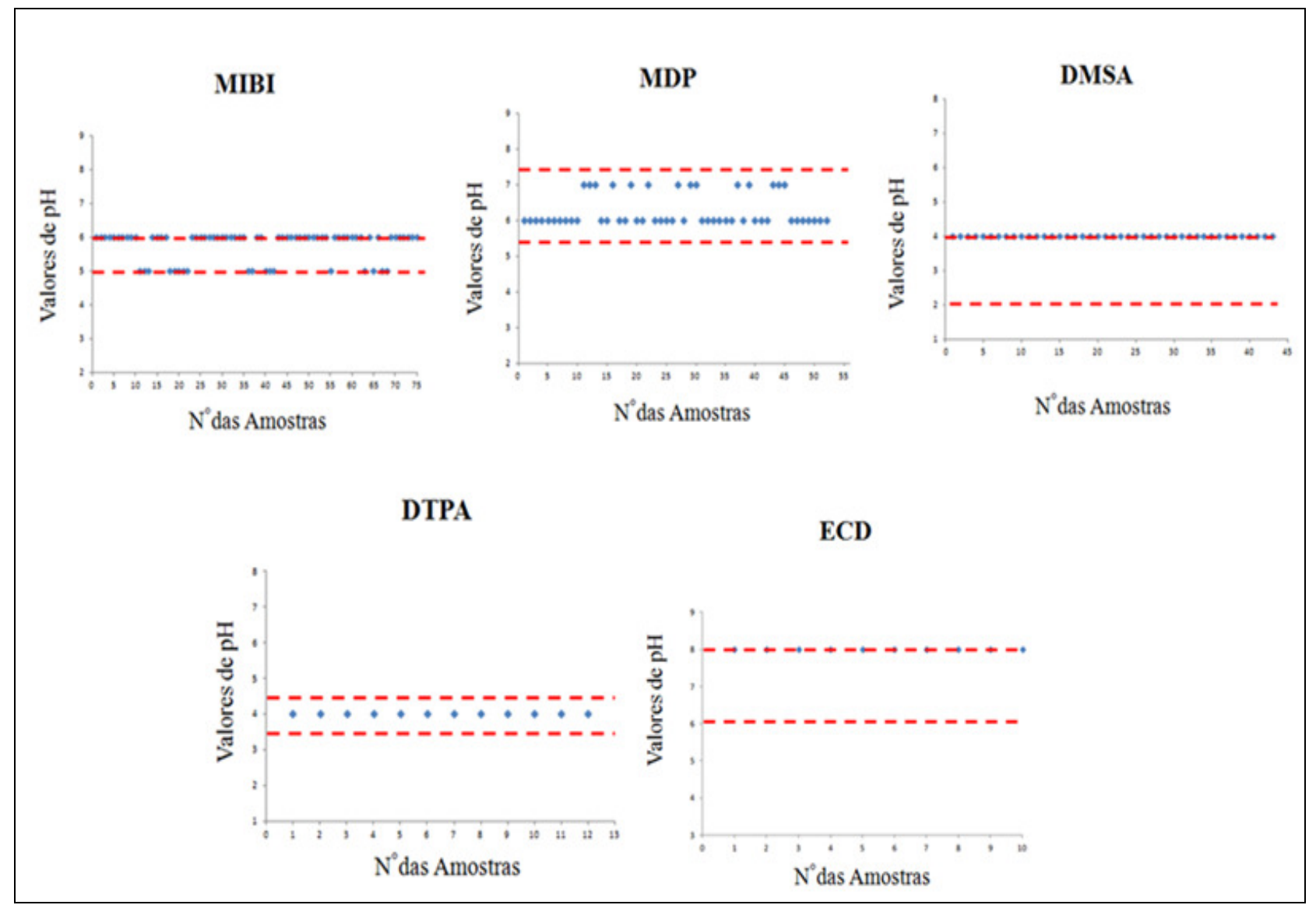

Os valores de $\mathrm{pH}$ das amostras dos 192 radiofármacos selecionados apresentaram-se dentro da faixa aceitável e recomendado pelos fabricantes, como descritos na Tabela 2. Apesar das 6 amostras de DMSA não terem apresentado uma eficiência de marcação ideal, elas não tiveram alterações fora do intervalo de $\mathrm{pH}$. Por se tratarem de moléculas com características ácidas, o DMSA e o DTPA apresentam $\mathrm{pH}$ predominantemente ácidos, e faixas de $\mathrm{pH}$ muito próximas (DMSA, entre 2,0 e 4,0; e o DTPA, entre 3,5 e 4,5) 


\section{CONCLUSÕES}

O SMN que segue os parâmetros de funcionamento e controle sanitário exigido pela RDC n 38/2008 garante a defesa da saúde dos pacientes, dos profissionais envolvidos e do público em geral. $\mathrm{O}$ estudo mostrou que o uso do método de cromatografia em camada delgada para avaliação rotineira do controle de qualidade é importante para identificar e evitar administração de radiofármacos de baixa qualidade em pacientes, evitando assim uma exposição de radiação ionizante desnecessária à pacientes e profissionais envolvidos na prática médica. Sugere-se, a implantação do CQ do eluato dos geradores de ${ }^{99} \mathrm{Mo} /{ }^{99 \mathrm{~m}} \mathrm{Tc}$ no SMN e sempre fornecer aos funcionários do SMN cursos de atualização e aperfeiçoamento abordando boas práticas de manuseio e fabricação de medicamento, além das normas de radioproteção. 


\section{REFERÊNCIAS}

1. ANVISA, Agência Nacional de Vigilância Sanitária. Resolução de Diretoria Colegiada n³8 de 04 de julho de 2008. [S.1.], 2008.

2. SAHA, G. Fundamental of Nuclear Pharmacy. New York: Springer, 2010.

3. ANDRADE, W. G.; SANTOS, P. A. L.; LIMA, F. R. de A.; LIMA, F. F. Utilização do Método CCD para Determinação da Pureza Radioquímica de Radiofármacos em Serviços de Medicina Nuclear da Paraíba e Rio Grande do Norte. In: II SIMPOSIO DE DOSIMETRIA INTERNA APLICADA A MEDICINA NUCLEAR. [S.1.]: DOSIMN,2011.

4. FARIA, D. P.; MARQUES, F. L. N.; YAMADA, A. S.; MIQUELIN, C. A. Avaliação dos custos para realização de controles de qualidade de radiofármacos marcados com $\left[{ }^{99 m}\right.$ Tc]tecnécio em serviços de medicina nuclear no Brasil. Radiologia Brasileira, p.47-51, 2011. 